\title{
Germanica
}

\section{«I.N.R.I. - La bonne nouvelle apportée par un pauvre pécheur » Le Nouveau Testament revisité par Peter Rosegger (1843-1918)}

«I.N.R.I. - Die frohe Botschaft eines armen Sünders», Das Neue Testament aus Peter Roseggers Sicht (1843-1918).

\section{Eva Philippoff}

\section{OpenEdition \\ Journals}

Édition électronique

URL : http://journals.openedition.org/germanica/2253

DOI : $10.4000 /$ germanica.2253

ISSN : 2107-0784

\section{Éditeur}

Université de Lille

\section{Édition imprimée}

Date de publication : 1 janvier 1999

Pagination : $97-115$

ISBN : 2-913857-00-0

ISSN : 0984-2632

\section{Référence électronique}

Eva Philippoff, « «I.N.R.I. - La bonne nouvelle apportée par un pauvre pécheur » Le Nouveau Testament revisité par Peter Rosegger (1843-1918) », Germanica [En ligne], 24 | 1999, mis en ligne le 31 janvier 2014, consulté le 06 octobre 2020. URL : http://journals.openedition.org/germanica/2253 ; DOI : https://doi.org/10.4000/germanica.2253

Ce document a été généré automatiquement le 6 octobre 2020.

(c) Tous droits réservés 


\title{
« I.N.R.I. - La bonne nouvelle apportée par un pauvre pécheur » Le Nouveau Testament revisité par Peter Rosegger (1843-1918)
}

\author{
«I.N.R.I. - Die frohe Botschaft eines armen Sünders», Das Neue Testament aus \\ Peter Roseggers Sicht (1843-1918).
}

Eva Philippoff

Et Jésus chante des Psaumes d'une voix claire ${ }^{1}$

1 On peut se poser la question de savoir pourquoi Peter Rosegger, autodidacte d'extraction paysanne modeste, et pourtant célèbre auteur d'histoires villageoises, a voulu écrire une Vie de Jésus. Fils de paysans montagnards pauvres, il était devenu à l'âge où il rédigea son roman biblique, en 1904, l'enfant adulé des Styriens, et au delà des frontières de la Styrie, de tous ceux, dans le Reich comme en Autriche, qui se réclamaient d'une seule Allemagne, germanique et unie. Vienne, la cosmopolite, la raffinée, considérait avec une condescendance bienveillante ses romans rustiques qui flattaient une classe moyenne encore proche de ses racines campagnardes, et qui étaient très loin des préoccupations de la littérature viennoise. Cependant on avait tort de ne pas prendre au sérieux les prises de position de Rosegger sur le plan politique et philosophique, car, même si elles manquaient de bases instruites, elles véhiculaient dans leur franc-parler d'«enfant du peuple", que Rosegger aimait à invoquer pour excuser son manque de vernis universitaire, bon nombre d'idées naissantes ou ambiantes sous une forme prégnante, grâce au ton et à l'imagerie populaires utilisés par Rosegger ${ }^{2}$.

2 Dans ce contexte et avec l'influence grandissante que Rosegger subissait des milieux pangermanistes de Graz dans les années quatre-vingt, milieux proches de l'Allemagne protestante, nous pouvons déjà imaginer que Rosegger, qui s'interdisait tout complexe 
d'infériorité sur le plan intellectuel, ait lui aussi voulu procéder à une interprétation des Évangiles, se créer un Jésus bien à lui.

3 Pour encore mieux saisir sa démarche, il faut aussi se souvenir que Rosegger, en dehors de son don pour la fabulation rustique, possédait une forte propension didactique, voire théologique et même prophétique, qui pouvait s'exprimer très librement dans le magazine mensuel édité et rédigé en grande partie par Rosegger, et qui s'intitulait «Der Heimgarten», que nous avons traduit par «La Veillée». Ce journal, prolongeant les anciens almanachs d'édification, parut de 1876 jusqu'à la mort de notre auteur et fut ensuite continué par son fils ${ }^{3}$. Nous disons aussi théologique, car pour Rosegger enfant, qui avait grandi dans une ferme isolée, située à une altitude de 1000 mètres, la première culture, ses premières impressions éthiques et esthétiques, comme celles d'un allemand non dialectal, provenaient de l'Église catholique et de son culte, très riche et très coloré dans ces régions. A douze ans déjà, l'enfant qui savait tout juste manier un peu la plume, composait des almanachs pieux, des sermons sur la pénitence, intitulés par exemple «Paroles adressées aux Chrétiens» («Einige Worte an die Christen») et «L'Honneur de Dieu » («Die Ehre Gottes»), ainsi qu'une Vie de Saint Joachim.

Son vœu le plus cher avait été d'accéder à la prêtrise - hélas, les moyens matériels manquaient, et les connaissances scolaires de Peter, péniblement acquises auprès d'un instituteur itinérant, étaient par trop lacunaires. Toute sa vie il sera hanté par ce regret qu'il exprime dans de nombreux récits ayant pour personnage central une figure de prêtre qui évolue généralement dans le milieu rural - celui que Rosegger connaissait ${ }^{4}$. Toutefois le passage du jeune homme à Graz à la fin des années soixante, au moment où fut aboli le Concordat et où débuta l'ère libérale, le contact avec des personnes issues du milieu libéral, devait fortement le marquer. Désormais il voit son idéal dans le "prêtre libéral», celui qui enterre les suicidés, marie les concubins, réconforte ses ouailles, plutôt que de les effrayer par les affres de l'Enfer. Plus tard, à l'époque où naquit le mouvement de Séparation d'avec Rome («Los von Rom») Rosegger va plaider pour un œcuménisme entre catholiques et protestants ${ }^{5}$, après avoir rejeté la tentation d'une conversion au protestantisme ${ }^{6}$, tentation à laquelle aucun de ses quatre enfants n'aura résisté. C'est ainsi que Rosegger croyait, au moment où il rédigea I.N.R.I., pouvoir concilier une collecte pour la construction d'une église protestante à Mürzzuschlag en 1900, avec une collecte pour la reconstruction de l'église de Sankt Kathrein, celle de son enfance, partiellement détruite par un incendie en 1904. Il est vrai que, discrédité aux yeux de l'Église par bon nombre de ses prises de position peu orthodoxes, il était considéré plutôt comme un renégat, et ne fut guère encouragé par le clergé local dans cette entreprise pourtant louable. Signe des temps: ceux qui donnèrent le plus furent les protestants allemands!

5 La préoccupation religieuse a donc été une constante dans la vie de Rosegger, et il était convaincu qu'il entretenait une «relation privilégiée » avec le «Bon Père au Ciel »" croyance aisément compréhensible quand on pense à la vie épanouie et heureuse qui fut celle de Rosegger: de l'existence obscure dans une campagne arriérée il était passé à la gloire d'un auteur dont les œuvres furent tirées à des millions d'exemplaires. Il faut pourtant mentionner un fait qui a probablement donné le véritable déclic à la rédaction de l'ouvrage biblique: en 1892 Rosegger, très malade, avait eu la vision du Christ apparaissant à ses côtés pour le réconforter : "Mince, vêtu d'une tunique bleu foncé [...], sobre [...], le visage émacié, la barbe naissante et la bouche expressive, malgré son silence ; les cheveux châtains simplement rejetés an arrière, et ses grands yeux bleus, 
pleins de grave douceur $»^{8}$. Nous y reconnaissons aisément le Christ des chromolitographies répandues à l'époque, une apparence extérieure que Rosegger va conserver pour la figure du Christ de son roman.

6 Une fois guéri, Rosegger passe ses heures claires à lire les Évangiles, et le Christ ne le quittera plus... « Je le vois à toute heure [...], à chaque fois que mon cœur pense à lui », nous confie-t-il. Et c'est cette conception visionnaire que Rosegger a conservée pour la rédaction d'INRI : il voit devant lui les scènes du Nouveau Testament, il en voit les protagonistes, les paysages de la Palestine biblique! Cependant il prend ses précautions : sachant sa démarche quelque peu téméraire, il se sert d'un intermédiaire, il choisit comme narrateur une tierce personne, un personnage suffisamment naïf, mais aussi suffisamment instruit pour rendre crédible sa démarche. Et il invente Konrad Ferleitner, condamné à mort pour un crime politique - il a commis un attentat contre un ministre - et qui attend l'accord, peu probable, de sa grâce. Il se voit alors en compagnie du Christ qui, tout en lui inspirant le livre, l'aide à traverser les semaines d'angoisse. Et Ferleitner prétend se référer à sa mémoire seule : ce sont « ses souvenirs d'enfance, les restes de ses connaissances scolaires, ce qu'il a retenu de certaines lectures, les images qu'il a vues, et surtout les légendes bibliques racontées par sa mère qui vont le guider $»^{10}$. Le lecteur est prévenu : nulle intention érudite, ni même historique ne préside à son entreprise. Ce qui en résulte est une sorte de "rêverie solitaire d'un chercheur de Dieu ${ }^{11}$, une fabulation très personnelle qui s'arroge le droit de procéder plus que librement avec les textes évangéliques, le plaisir de se raconter son Christ, celui qui est toujours près de lui (de Ferleitner comme de Rosegger), qui lui " permet tout $»^{12}$.

7 Nous verrons néanmoins que la démarche de notre auteur n'est pas aussi naïve qu'elle semble $^{13}$, car il dispose les matériaux bibliques d'une façon qui trahit des choix délibérés, des choix qui dépassent de loin ce dont pourrait se souvenir un condamné à mort dépourvu de tout support écrit. En effet, - et Rosegger insiste là-dessus l'aumônier, en catholique obéissant, refuse de confier les Évangiles au criminel pénitent, pour lui apporter à la place une œuvre d'édification et de contrition, telle que la « Description de la Vie du Christ, de sa Mère Marie et d'un grand nombre de Saints un Trésor spirituel du Père Cochem $»^{14}$, ouvrage que le jeune Rosegger avait déjà rejeté comme étant peu apte à apporter la consolation à ceux qui doivent affronter la mort. Et voilà que Ferleitner procède comme le «Schulmeisterlein Wuz» de Jean Paul, le petit instituteur de campagne, qui, à défaut de pouvoir acheter des livres, s'en fabrique luimême : il réécrit l'Histoire Sainte.

Cependant, nous le disions déjà, le Christ de Ferleitner-Rosegger n'est pas celui des Évangiles, ni celui qu'il a pu connaître par les sermons entendus le dimanche. C'est un Christ édulcoré, humanisé, rapetissé, ramené à taille humaine, un Christ chez qui sont expurgées les rugosités tranchantes de certaines paroles. Le Christ de FerleitnerRosegger est un homme bon, moralement grand, car détaché des biens terrestres et prêchant l'amour du prochain. C'est le "Fils préféré de Dieu »" un Christ de pure bonté qui, s'il exige un amendement, un retour sur soi, incite tout au plus à une vie simple, sans excès, ni dans la recherche des biens, ni dans celle des plaisirs. C'est un Christ des plus rationnels, car les miracles sont, ou bien escamotés - aucun démon n'est mentionné ni chassé - ou bien «rationalisés » : la multiplication des pains s'explique par le désir de chacun des disciples de laisser à l'autre l'avantage du seul pain qui reste et qui fait alors la ronde sans être mangé par l'un des douze ni par le Christ... La pêche 
miraculeuse avec son lourd contenu symbolique - ou à cause de lui ? - n'est tout simplement pas mentionnée; Saint Pierre est dispensé de la marche sur les eaux, alors que Jésus «apaise " les tempêtes en les contemplant et en attendant tranquillement qu'elles se calment, (pp. 93 et 140) Puisque Rosegger emprunte à l'Évangile selon Saint Jean, en dehors des topos consacrés de la christologie du logos, la fiction de Jean, disciple préféré de Jésus (il en est même un cousin chez Rosegger !), il est clair qu'il ne pouvait ignorer l'épisode de la résurrection de Lazare. Mais, comme pour d'autres guérisons opérées par Jésus, il l'enveloppe dans une tournure narrative au passé, passablement ambiguë ${ }^{16}$, et c'est ainsi que Lazare, frère de Marie-Madeleine - alors que Marthe est sa mère, la femme de son hôte à Béthanie (!) - se contente de s'écrier du bout de la table: «Tu m'as apporté la résurrection!» «Et à moi - plus que cela!", renchérit Marie-Madeleine ${ }^{17}$.

Or, tout en se montrant peu enclin à accepter le merveilleux biblique, Rosegger s'arroge le droit à une grande liberté de fabulation qui, certes, puise souvent dans les légendes populaires provenant des sources apocryphes, mais qui prend parfois des aspects fort personnels et curieux. C'est ainsi que Rosegger dote la mère du Sauveur d'un visage très réel, dépassant de loin les quelques caractéristiques conférées par Luc ou par Jean, mais qui rappellent en revanche très fortement la propre mère de Rosegger! Marie, mère de Jésus, accompagne chez Rosegger toute la vie de son fils de ses admonestations et de ses gages d'amour, elle est - et nous retrouvons là Saint Jean - présente sous la croix, où elle voit dans une sorte d'arbre de Jessé élargi et fantaisiste, défiler en direction du Paradis, Adam, Abel et Caïn, bras dessus, bras dessous (!), Abraham et sa progéniture mâle, Salomon et David (dans cet ordre), Isaïe et Jérémie, Joachim et Anna, ainsi que Joseph qui l'attend en chemin...

10 La Vierge, sous la plume de Rosegger, conseille également à son fils, quand il rencontre la réprobation de leur entourage à Nazareth, d'abandonner sa quête spirituelle, de rester, honnête menuisier, auprès des siens, de ne pas troubler la foi de ses congénères : "Laisse les gens trouver leur bonheur comme ils l'entendent. S'ils sont venus à Abraham (!) jusqu'alors, ils continueront à trouver le chemin vers lui-même sans toi ${ }^{18}$.

11 Rosegger reflète ici très exactement la situation qui fut la sienne quand, revenu de Graz dans son village natal, il fait montre de son vernis intellectuel, citadin et libéral, et que le curé du village l'invite vigoureusement à faire amende honorable, à quitter ce " chemin dangereux ». Dans un roman autobiographique, Rosegger montre même ses parents chassés ignominieusement du village natal à cause de l'« hérésie » du fils ! ${ }^{19}$

12 Ces petits dérapages vers le milieu familier styrien émaillent également le champ sémantique, quand Rosegger emploie, dans l'admonestation de la Vierge adressée à son fils et citée plus haut, l'expression «ohne Deiner» («sans toi »), au lieu de «ohne dich», quand il parle de l'odeur "de renfermé et de poussière moisie qui se dégage des vêtements des disciples» (p. 208), quand il dit que Joseph retourne à Bethléem, son «Heimatstädtl» (p. 40), quand la Galilée est décrite comme "gebirgig» (p. 38 et 98), et le Mont Hermon comme un sommet des Alpes avec des « déserts de glace («Eiswüsten») et des parois abruptes («starrendes Gewände») où l'on entend le « tonnerre des torrents » («donnernde Gebirgsbäche») (p. 235).

13 Ces petits glissements vers le style familier n'ont rien de déplaisant, on est davantage troublé quand Rosegger affuble les docteurs de la loi à Jerusalem de longs kaftans noirs et de bonnets en velours ${ }^{20}$, quand il prétend que la foule veut aux Rameaux mener Jésus au « Palais des rois » (p. 267), quand il présente le maître de la maison à Béthanie, où 
Jésus retrouve Marie-Madeleine dans un fauteuil roulant (p. 287). Mais on reste carrément interdit quand Rosegger, au lieu de «Pharisäer» et «Rabbiner» parle de «Phariten» et «Rabbiten», quand il attribue aux disciples des métiers comme «Kanner» (sic), «Riemer et Kahner» ${ }^{21}$, quand il interpole l'histoire de Joseph en Egypte dans l'histoire de l'enfance du Christ et qu'il fait séjourner celui-ci à la cour du Pharaon (p. 78 sq.), quand il fait vendre aux enchères à l'infâme Juif Schobal la tunique du Christ crucifié, quand il interprète l'inscription portée sur la croix par Ponce Pilate comme pouvant signifier : «In Not ruf ihn» (« Appelle-le dans la détresse »), ou «Jesus'Nähe rettet ihn» («la proximité du Christ le sauve »; il est question du larron repentant), ou encore «Im Nirvana ruh'ich» («Je repose dans le nirvana ») (p. 327 et 334)! Sans oublier que Rosegger écrit avec une obstination irritante «pfählen» (empaler) à la place de «kreuzigen», crucifier...

Mais regardons de près ce " $5^{\mathrm{e}}$ Évangile ", comme une critique viennoise a très bien nommé ce livre que, selon un autre écho, «Ferleitner n'a pas pu écrire et Rosegger n'aurait pas dû écrire $»^{22}$.

Pour le début, aucune fantaisie. Rosegger suit l'Évangile selon Saint Luc et fait naître le Sauveur à Bethléem, le «Heimatsstädtl» de Joseph, descendant de David. Joseph aime parfois parler de son illustre ancêtre, et ceci sans fausse honte, car lui aussi a commencé petit, il était pâtre avant de tuer Goliath et devenir roi... Tout se passe comme Saint Luc le raconte. Marie accouche dans une étable appartenant à un berger nommé Ismaël - mais qu'importe si Jésus naît chez un descendant de la branche arabe, Rosegger voulait de toute façon peindre un Christ œcuménique, et la différence entre gentils et juifs ne l'intéressera pas, du moins en ce qui concerne la prédication du Christ et le choix de ses destinataires.

Les Trois Mages légendaires en revanche se résument en un seul: Balthazar, et sa provenance est précise : il vient des "jardins embaumés de l'Inde "23; guidé par une constellation d'étoiles qui inscrit dans le ciel les lettres I.N.R.I., Balthazar se met en route vers l'ouest et trouve auprès de l'enfant dans l'étable, comme suspendu en l'air, le message « Vous serez bienheureux, vous serez éternels $»^{24}$. Rosegger fait intervenir ici la philosophie hindoue qui prévoit la métempsycose et le nirvana comme ultime récompense, philosophie qui préoccupait Rosegger à l'époque à tel point qu'il imaginait, dans sa métaphysique personnelle, une sorte de perfectibilité à travers les générations aboutissant finalement à l'être parfait ${ }^{25}$. Le nirvana, le néant, que Rosegger envisageait comme alternative à la Vie Éternelle annoncée par le Christ, sera rejeté, "La non-existence, cela n'existe pas ${ }^{26}$, se fait dire par l'apôtre Jacques le vieillardermite en quête de nirvana, que Rosegger introduit obstinément à des moments divers de son récit. (Nous nous rappelons qu'une des interprétations de I.N.R.I. consistait à dire « Je repose au nirvana »).

Mais revenons à la vie du Christ. Inutile de dire qu'il n'y a chez notre auteur pas la moindre allusion à la Conception Immaculée de la Vierge, et que Joseph, en bon père, prend soin de sa petite famille qu'il emmène en Égypte pour fuir la persécution d'Hérode, (qui est mort 4 ans avant la naissance du Christ, mais puisque le Christ est probablement né 4 ans avant notre ère, il y a des chances qu'ils fussent contemporains pour peu de temps). En route pour l'Égypte, nous rencontrons, avec la Sainte Famille, pour la première fois le tandem redoutable de Barabbas et Dismas, brigands de grands chemins, installés dans le désert de Judée et ailleurs. Le sort va les frapper en même temps que le Christ, car Barabbas est bien sûr l'un des deux larrons crucifiés avec le 
Sauveur. Dismas sera le second, celui qui se repent et à qui Jésus promet le ciel avant le soir. Il est vrai que Dismas aura eu le mérite de livrer aux autorités romaines Barabbas et sa propre personne. Mais avant d'en arriver là, Rosegger leur consacre de larges scènes où, entourés de leur bande de brigands, on les voit à l'œuvre, dans le décor splendide du désert, paysage que Rosegger, à l'instar de Karl May, grand peintre d'aventures au Kurdistan, décrit brillamment sans l'avoir jamais vu.

Or, la Sainte Famille sur son trajet vers l'Égypte, fait une autre rencontre digne d'être mentionnée : une jeune femme laide, brune et hirsute qui, faisant passer son propre fils pour une fille, détourne les foudres des sbires d'Hérode vers le petit Jésus qui vient de passer devant elle. Et ce fils, épargné grâce à ce mensonge ne sera autre qu'Asuérus, le Juif errant, l'ignoble cordonnier qui refuse au Christ portant sa croix un instant de répit au seuil de sa porte. Épisode que Rosegger reprend à la légende avec une certaine complaisance. Et voilà encore notre vieillard entiché de nirvana, revenu du désert pour jeter l'anathème sur cet enfant: "C'est en toi que la malédiction de ton peuple se réalisera, Juif sans cœur ! $»^{27}$

Tout au long de l'épisode de la Fuite en Égypte, le récit de Rosegger est placé sous le signe de nombreuses réminiscences de l'Ancien Testament : en route, en passant par le Mont Sinaï, Joseph n'omet pas de se tailler un bâton dans le buisson ardent, et ce même bâton se mettra à fleurir au moment où la Sainte Famille arrive sur le sol égyptien. Ici l'allusion au bâton de Moïse par lequel Dieu persuade celui-ci de mener son peuple hors d'Egypte est évidente (Exode 4/1-5), et Rosegger se souvient aussi que le bâton d'Aaron fleurit pour le désigner comme chef parmi les douze tribus. (Nombres 17/16-26). Pour rendre le parallèle encore plus éloquent, Rosegger insère même une traversée de la Mer Rouge - dans le sens opposé cette fois - et moins grandiose que celle de l'Ancien Testament. Toujours est-il que Joseph et Marie disparaissent devant les yeux de leurs poursuivants arrivant sur la lande (!), et pour ne pas être soupçonné d'avoir laissé passer un miracle, notre évangéliste-rationaliste insinue qu'ils ont probablement pris une barque de pêcheurs...

En Égypte, le fil de la narration suit largement l'histoire de Joseph et ses frères de l'Ancien Testament. D'abord nous apprenons que notre Joseph, père et nourricier, fait vivre sa famille très honnêtement en fabriquant des paniers, le bois étant rare en Égypte. Un jour l'idylle à trois est pourtant troublée par le Pharaon qui remarque l'enfant qui se distingue par sa grâce et sa beauté, et il l'invite à venir vivre dans son palais. Le petit Jésus s'y rend de bonne grâce - (contre le vœu de sa mère, qui entame une longue série de séparations d'avec son fils) - pour pouvoir y «étudier les vieux parchemins » et pour consoler le Pharaon par sa parole déjà divine, selon laquelle le maître de l'Égypte pourrait être guéri de sa mélancolie, s'il suivait tout simplement le précepte de faire briller à l'intérieur la lumière qui l'entoure à l'extérieur : «Wende es um, wende das Licht nach innen!» (p. 86)

21 Mais le Pharaon n'est pas le seul à qui le petit Jésus dispense sa sagesse, déjà grande : il administre également une leçon à un vieillard-ermite, caché au fond d'une pyramide, qui étudie nuit et jour les hiéroglyphes pour trouver le nirvana (!)... D’abord Jésus libère la chèvre confinée avec le vieillard dans la grotte, ensuite il lui réclame une sagesse " pour les vivants ", et non pas " pour les morts ", pour finir par une chiquenaude, en répondant au vénérable vieillard qui veut tirer son horoscope, qu'il est né «sous le signe du bœuf et de l'âne » et que son père est celui qui « dirige les astres » (p. 89). 
le temps est venu pour ramener la Sainte Famille sur la terre natale. Les persécutions par Hérode ayant cessé, Joseph décide de ramener les siens. Cette fois, on va emprunter la voie maritime par la Méditerranée, et en prenant un bateau au vu de tout le monde. Néanmoins, cette traversée sera pour notre auteur l'occasion de broder sur une première tempête que le jeune Jésus apaise - en contemplant avec ravissement les flots déchainés, qui finissent par s'apaiser sans grand dommage pour le bateau (p. 92/93).

Avant de rentrer à Nazareth - Rosegger suit ici Saint Luc et non pas Saint Matthieu, pour qui la Sainte Famille n'est pas originaire de cette ville - on décide de visiter Jérusalem pour se recueillir sur les lieux saints. Et c'est ici que les parents désolés perdent leur fils et le retrouvent finalement - toujours en suivant Saint Luc - prêchant dans le temple.

Dès maintenant nous pouvons observer comment Rosegger procède pour relever dans l'enseignement $\mathrm{du}$ Christ les paraboles qui s'accordent avec sa vue très personnelle $\mathrm{du}$ Christ, et qu'il en élimine d'autres, celles qui pourraient prêter à confusion ou qui sont peu claires ${ }^{28}$. Pour intégrer le plus grand nombre de paraboles - Rosegger veut être exhaustif - et toujours dans un souci de vivacité, il prend ce qui lui convient dans la logique du récit, sans le moindre souci de l'ordre observé par les synoptiques, chez qui une certaine évolution psychologique du Christ n'est pas absente. Entre le Sermon sur la Montagne, l'envoi des Douze, la Transfiguration et l'entrée à Jérusalem, la prédication du Christ va d'une parole confiante, aimante, à un durcissement parfois presque haineux, en passant par une conviction de plus en plus intime de sa filiation divine. Le Christ de Rosegger, en revanche, est un homme, certes un homme d'exception, mais qui reste toujours le même être, fait de bonté et d'amour, et dans le caractère duquel Rosegger intègre comme à contre-cœur, par quelques touches, les imprécations finales envers Jérusalem.

Donc, non seulement les parents de Jésus retrouvent leur fils alors qu'il enseigne au Temple, Rosegger profite de l'épisode pour placer tout de suite quelques paraboles que les Saintes Écritures placent nettement plus loin. Ici il s'agit de la prédication contre l'observation trop stricte du sabbat, illustrée par l'épisode des épis arrachés par les disciples (Mt 12/1-8, Mc 2/23-28) et celui de la brebis tombée dans un puits (Mt 12/11).

De retour à Nazareth, Joseph reprend possession de sa maison où un cousin du nom de Nathanaël (ce ne sera pas le disciple!), s'était installé en son absence, et commence à enseigner son métier à son fils. Nous apprenons aussi que la Vierge fait de la couture pour les femmes du village (p. 101). Probablement dans le souci de rester fidèle sur ce point à Saint Jean, Rosegger voit Jésus unique enfant du couple, contrairement aux synoptiques qui laissent entendre qu'il aurait eu des frères et sœurs.

Jésus est un garçon sérieux, obéissant, il n'a qu'un seul défaut : c'est l'inattention dans son métier. Inattention qui vient du fait qu'il est porté sur la méditation. «Il ne pense pas, cela pense en lui $»^{29}$, nous explique Rosegger, qui veut nous dépeindre un garçon doué d'inspiration supérieure, mais néanmoins pas en dehors de la sagesse de son peuple, car, contrairement aux synoptiques, il insiste pour dire que le jeune Jésus collecte dans son entourage les parchemins des Livres Saints que les Galiléens, mauvais Juifs, comme on sait, lui cèdent volontiers (p. 101). Ici Rosegger prête encore une fois au Christ des traits de sa propre jeunesse : lui aussi fut un garçon "inspiré », absent dans son apprentissage de tailleur qui précéda son départ pour Graz, un garçon qui collectait pieusement les livres que certaines - rares - personnes de son entourage pouvaient lui 
céder. Et c'est ainsi que, tout comme le jeune Peter, Jésus s'attire la réprobation des gens de son village. Il est trop prompt à expliquer les textes sacrés, trop différent, trop contrariant ! Et Rosegger illustre ce dernier point par la parabole de la vraie pureté (Mt 15/10-20, Mc 7/14-23), et par celle que Saint Luc insère peu avant la Passion (Le 21/1-4 et Me 12/41-44) : la générosité de la pauvre veuve, en opposition aux riches pharisiens. Détail amusant : à la place du Trésor où la veuve verse son obole, Rosegger parle du tronc d'église («Opferstock») bien de chez nous (p. 108).

Nous voyons donc comment Rosegger opère parmi les paraboles le choix le mieux adapté à sa démonstration, sans le moins du monde tenir compte de l'ordonnance adoptée par les Évangiles.

Parmi les épisodes marquants, il y en a un que Rosegger ne pouvait omettre : les Noces de Cana (Jn 2/1-12), miracle que Saint Jean place au début du ministère du Christ. Ici et c'est l'unique exception - Rosegger renonce à donner une explication rationnelle au miracle. Le Christ, agacé par les mondanités de la noce, aurait simplement dit, quand il apprit qu'on manquait de vin: "Qu'ils prennent donc de l'eau! $»^{30}$, ce qui fut fait. Ensuite il constata avec les autres et à son grand étonnement qu'à la place de l'eau il y avait du vin dans les récipients et il ne lui restait qu'à remercier Dieu: "Si c'est Ta volonté de transformer l'eau en vin, on verra peut-être aussi du vin nouveau dans les vieilles outres (!), et l'esprit et la puissance de Dieu dans les lettres mortes ». Jean, le proche parent, qui est avec lui, atteste : « Tu dois être du Ciel ! » ${ }^{31}$ )

Jusqu'ici il n'a pas été question de Saint Jean-Baptiste. Il apparaît chez Rosegger au $10^{\mathrm{e}}$ chapitre (non numéroté) de son récit biblique, prêchant dans le désert, baptisant «depuis le massacre des Saints Innocents » (p. 114). De ce fait Rosegger abandonne sa fidélité à Saint Luc et l'allusion à la grossesse concomitante d'Elisabeth et de Marie. S'il suit par ailleurs assez fidèlement le récit concernant le Précurseur, il commence à introduire ici une touche de misogynie qui se manifestera tout au long de son livre à travers plusieurs anecdotes, qui sont, bien sûr, totalement absentes des Écritures. C'est ainsi que l'austère prédicateur refuse le baptême à un groupe de femmes à la pénitence quelque peu coquette, et les chasse par ces paroles vigoureuses : « Vos droits, vous vous les arrogez toutes seules, et vos devoirs, on vous les donne. Le commandement de la femme (Weib!) est: «Tu ne seras point adultère! $»^{32}$. Bien évidemment, Rosegger raconte avec une délectation certaine la mort de Saint Jean-Baptiste qu'il enchaîne directement après le baptême du Christ, et, pour ne pas avoir à y revenir, il fait immédiatement chasser Hérode qui tombe dans les mains d'Arétas, son beau-père bafoué33. Très vite notre auteur fait alors intervenir la parabole de la femme adultère et même sous une forme dédoublée. Il intercale seulement deux épisodes : la tentation du Christ dans le désert où c'est Barabbas, «Roi du désert », qui lui propose ses loyaux services pour devenir « maître du monde », ainsi que les prémisses de la conversion de Simon-Pierre, avant de nous faire rencontrer la première des pécheresses, celle à qui Jésus pardonne, et qui est Marie-Madeleine (Le 7/36-50 et Jn 8/1-11), mariée, chez Rosegger, depuis peu au «brave, vieux Jobsohn » qu'elle trompe avec « un jeune fat » ${ }^{34}$. Après avoir été sauvée par le Christ des mains d'une foule haineuse, elle devient une de ses plus ferventes adoratrices - à ce point qu'elle juge plus décent, un peu plus tard, de se retirer du groupe des fidèles, auprès de son parent à Béthanie, le mari de Marthe, père de Lazare, que nous connaissons déjà...

31 Mais le contentieux de Rosegger avec les femmes ne semble pas encore réglé. D'abord, il double le sauvetage de Marie-Madeleine par un épisode parallèle où la pécheresse 
non repentante n'est pas exaucée - elle était celle qui criait le plus fort contre Marie de Magdala! Et ensuite, Rosegger se met à insérer une figure féminine à un endroit inattendu: il invente une épouse - une mégère du nom de Judith - au publicain Matthieu-Lévy, ce qui expliquerait pourquoi cet homme n'eut aucun mal à quitter son foyer pour suivre séance tenante le Christ, (p. 155 sq.). Pour l'adhésion de Simon-Pierre - qui ne fut pas moins prompte - il fallait argumenter autrement. Simon est un indécis, un rêveur qui, malgré l'amour que lui porte la charmante Beka, ne peut se décider à l'épouser. Il n'a pas encore trouvé sa voie, il cherche, et quand le Christ arrive avec Jacques et Jean lui demander de le transporter dans sa barque, sur le lac de Génézareth, il se joint à eux.

Le sort que Rosegger réserve à la pêche miraculeuse et à la tempête apaisée a déjà été mentionné; les autres miracles - parmi eux la guérison de la fille de Jaïre, le paralytique de Capharnaüm, le jeune homme de Naïn, ainsi que plusieurs autres guérisons - Rosegger les situe soigneusement dans le passé, les fait raconter par ouïdire, mais se garde d'en décrire aucun. En revanche, il s'arrange pour placer avant le sermon sur la montagne, événement-clef de la prédication du Christ, un certain nombre de paraboles, dans un pêle-mêle qui s'explique moins par la mémoire défaillante de Ferleitner - elle est au contraire trop bonne! - que par l'utilitarisme narratif de Rosegger. C'est ainsi qu'à la femme adultère succèdent les paraboles du bon berger (Jn 10/1-21), du fils prodigue (Le 15/11-32), du pharisien et du publicain (Le 5/27-32 et Mt 9/9-13), pour finir avec Saint Luc 16/13 et Mt 6/24: On ne peut suivre deux maîtres.

En dehors du sermon sur la montagne que Rosegger suit assez fidèlement, tout en l'édulcorant et en le plaçant sous la devise "Il est un Bon Père au Ciel », Rosegger ajoute un grand chapitre dédié à l'instruction du Christ, où il insère un grand nombre de paraboles qui ne pouvaient être absentes d'une œuvre dont les ambitions dépassent largement celles annoncées dans le récit-cadre. Il s'agit essentiellement des paraboles que Saint Matthieu rapporte dans le chapitre 13: celle du semeur, "Il faut rester vigilant ", «l'ivraie et le blé », «le grain de sénevé », «le levain », « le trésor dans le champ » et "la perle ». Logique avec lui-même et n'ayant pas mentionné la pêche miraculeuse, Rosegger omet la parabole du filet (Mt 13/47-50). Suivent encore les paraboles de Mt 18/21-35 « les serviteurs et débiteurs », Mt 20/1-16 « les ouvriers dans la vigne ", Mt 22/1-14 « les invités au repas du roi », Luc 16/1-19 "l'économe infidèle ", Mt 21/28-32 «les deux fils aux champs » pour revenir à Mt 22/14 : «beaucoup sont appelés, peu seront élus ».

Entre temps Rosegger a introduit dans son récit deux personnages dont l'un n'est nullement mentionné dans les Évangiles : il s'agit de Judas Iscariote et de Saül-Paul, apôtre des gentils. À Judas, Rosegger essaie de donner le visage du juif un peu borné et avare, qui se pend, ahuri par sa propre trahison, alors que Saiil, le «farouche tisserand $»^{35}$, croise à plusieurs reprises le chemin des disciples, les menaçant du haut de son cheval noir, entouré d'un groupe de lansquenets (!), avant de les supplier de pouvoir faire partie de leur groupe (p. 232 et 345).

La composition la plus étonnante dans laquelle Rosegger s'est hasardé est pourtant celle qui concerne Simon de Cyrène. Ce personnage épisodique qui aurait, selon les synoptiques, porté la croix du Christ, est amalgamé par Rosegger avec le «jeune homme riche » (Mt 19/16-30, Mc 10/17-31, Le 18/ 18-30) qui cherche le "royaume de Dieu » et à qui le Christ conseille - vainement - de tout abandonner pour le suivre. Chez 
Rosegger, ce futur porteur de la croix intervient au milieu du récit, offrant à notre auteur l'occasion d'une fresque colorée, où il décrit la caravane richement dotée par laquelle Simon, désireux de trouver la vie éternelle, se fait transporter dans le désert, à la rencontre du prophète nazaréen. La prédilection juvénile de Rosegger pour les fastes de l'Orient s'exprime ici dans la description du campement luxueux de Simon. Bien évidemment, le conseil de pauvreté que lui donne le Christ, est rejeté, mais l'impénitent riche sera puni sans délai par ces autres habitants du désert que nous connaissons déjà : les brigands Barabbas et Dismas qui le dépouillent de ses biens, mais lui laissent la vie, grâce à l'intervention du déjà modéré Dismas. La fameuse parole de Mt 19/24 : « II est difficile à un chameau de passer par le chas d'une aiguille, mais il est encore plus difficile à un riche d'entrer dans le royaume de Dieu ", ne manque pas d'être citée par Rosegger, mais préférant la logique à l'hyperbole, il met à la place du « chameau » « un poil de chameau »! ${ }^{36}$

Il est clair que Rosegger, sous le prétexte de laisser parler un certain Ferleitner, procède à une appropriation sans scrupules de l'Histoire Sainte, dans un récit où, en effet, le «Christ permet tout ». Peu importe alors si, lors de la Transfiguration, il n'est nullement question ni de Moïse ni d'Eue, si Thaddée, faisant honneur à son nom («Thaddädl» en allemand dialectal est un niais, un guignol), fait le pitre (p. 160), si, pour l'entrée à Jérusalem les disciples cherchent un cheval destiné à leur maittre et se contentent ensuite d'un âne ${ }^{37}$, si les disciples sont présents à la mise en croix, et si c'est la Vierge qui découvre le caveau vide (p. 338). On serait tenté de penser que Rosegger s'est dit ce que Ludwig Anzengruber, son compatriote et ami, a lancé à ceux qui se scandalisaient en 1870 du Dogme de l'Infaillibilité, énoncé par Pie IX : « Si vous avez cru le tout jusqu'alors, vous pouvez accepter une once de rajout $! »^{38}$. En effet, qu'importe qu'il y ait un peu plus ou un peu moins de fantaisie, du moment qu'il y a un «Bon Père » au ciel qui nous garantit une vie éternelle, on est libre de s'imaginer la vie du Christ comme on veut! Le livre de Rosegger, n'est-il pas l'aveu implicite qu'il n'accorde aucune foi aux Évangiles comme textes révélés? Que ce sont pour lui des récits comme d'autres, des récits où l'on aurait tourné un certain nombre de faits plus ou moins historiques de telle où telle façon? N'est-ce pas l'aveu que le travail fait depuis le siècle des Lumières, par Kant, Herder, Schleiermacher, David Friedrich Strauss, Renan et d'autres a entièrement porté ses fruits? En effet, une fois admis que les Évangiles appartiennent à la fiction théologique, chacun peut broder à sa façon, ce qui, en fin de compte, a abouti il y a peu à la version humoristique des Mounty Python «la Vie de Brian", un film qui peut choquer, mais qui me semble plus honnête dans son incrédulité iconoclaste qu'un ouvrage comme celui de Rosegger prétendant à une authenticité nourrie de la foi, dont seule la mauvaise mémoire de Ferleitner aurait enfreint la correction orthodoxe! La feinte narrative ne tourne-t-elle pas à l'hypocrisie? Rosegger n'avait pas le courage de dire: "Je vais vous raconter une histoire de Jésus comme moi je l'imagine », car il aurait été obligé d'affronter l'avis non seulement des hommes d'Église mais encore des chercheurs sérieux qui l'auraient probablement taxé de fantaisiste, d'iconoclaste, d'infantile, etc. Pour se soustraire à cette contrainte et à un jugement probablement négatif, et pour écrire quand même son "Jésus - homme populaire ", il a très astucieusement inventé un récit-cadre inattaquable. Ce qui est grave dans cette entreprise, c'est que cette œuvre - qu'on pourrait par moments soupçonner d'intentions humoristiques - a été parfaitement prise au sérieux par la très grande communauté de ses lecteurs fidèles, et aurait même servi de "guide spirituel » selon le dire de certains. Ce qui montre à quel point un 
public petit bourgeois, peu instruit, s'en laissait conter, pourvu qu'on le rassurât à bon compte sur le salut de son âme. Une attitude athée, ou du moins agnostique, comme celle d'Anzengruber, nous aurait semblé plus honnête, plus courageuse, mais aussi plus malcommode. Ce Christ, brave garçon, promettant inlassablement la Vie Éternelle pourvu qu'on fasse un petit effort, est le dernier avatar d'un processus de dissolution, de dislocation de la religion chrétienne au seuil de la Première Guerre mondiale. Il est un autre signe de l'hypocrisie morale du temps, qui fut précisément un des facteurs qui ont conduit au grand holocauste. Rosegger, qui se voulait visionnaire, prophète, n'aura finalement été qu'un propagateur inconscient d'idées déjà éculées.

\section{NOTES}

1. - Peter Rosegger : Gesammelte Werke, Staackmann, Leipzig, 1913-1916.

Edition définitive en 40 volumes (nous citerons ED) INRI est le vol. 38, ici nous citons p. 106.

2. - C'est ainsi que l'idéologie nazie a pu trouver dans l'œuvre du poète styrien maint trait typique, comme par exemple, l'anti-intellectualisme, la mise en avant de la vie près « du sol », la haute considération de la race germanique, « seule créatrice de valeurs authentiques ».

3. - A partir de 1910 c'est son fils Hans-Ludwig Rosegger (1880-1929) qui en assurait la direction.

4. - Par exemple dans les nouvelles : «Der Dorfkaplan» (1869), «Johannes der Liebling» et «Maria im Elend» (1875), «Die Mutter in der Grotten» (1883), le personnage de Einspänig dans le roman «Die Schriften des Waldschulmeisters» (1875), les romans «der Gottsucher»1883, «Das ewige Licht» (1897) et «Die beiden Hanse» (1912). Cf. également le titre d'un recueil d'admonestations : «Bergpredigten» (1885). Pour sa propre vocation ratée il dit p. ex. : «Meine Neigung fur diesen Stand ist nicht umzubringen, und ich bedaure immer, daß mir einst die Wege dazu nicht offen gestanden.» (ED 40 p. 116).

5. - Cf. la nouvelle «Christ auf der Heide» 1900.

6. - Les volumes «Allerlei Menschliches» de 1892, «Mein Himmelreich» (1901) et «Das ewige Licht» (1897) rendent largement compte de ces préoccupations.

7. - ED 5 p. 67 : «Gott wird es so einrichten, daß seine Menschenkinder immer was haben zur Freude...» ou : ED 34 p. 333 : «Wer es mit Gott zu tun hat, [...] der (schreitet) Arm in Arm mit Gott...», il est souvent question chez Rosegger du « braver Himmelvater».

8. - ED 34/53 : «Schlank, in seinem dunkelblauen Gewände [...] schmucklos [...] ein schmales Antlitz mit jugendlich sprossendem Barte und dem schweigend beredten Mund, mit schlicht zurückgelegtem, dunklem Haar und mit seinem großen blauen Auge voll milden Ernstes.»

9. - Ibid.

10. - ED 38 p. 34 : «Aus kindlichen Erinnerungen, aus den Resten des Schulwissens, aus den Bruchstücken seiner Bücherbelesenheit, nach gesehenen Bildnissen, vor allem aus den biblischen Erzählungen der Mutter, will er es wagen, den Herrn Jesus so lange zu bitten, bis er kommt».

11. - Cf. son roman «Der Gottsucher» de 1883.

12. - Kienzl-Rosegger: Briefwechsel, éd. par Hans Sittner, Vienne, 1953, p. 341 : «Wenn Christus nicht wäre. Der erlaubt mir alles [...] Christus sagt mir [...] sei du selbst.»

13. - Nous savons que Rosegger a lu «La Vie de Jésus » de Renan dans la traduction allemande, à son arrivée à Graz, et que cette lecture lui causa un grand désarroi. Il n'est pas exclu qu'il connaissait aussi l'œuvre de David Friedrich Strauss et qu'il s'intéressa à d'autres publications 
concernant la vie du Christ, comme p. ex. : «Les paroles du Christ» de Houston Stewart Chamberlain qu'il avait lu peu de temps avant la rédaction de INRI. On sait aussi qu'il fut impressionné par la série de conférences que le Suisse K. Furrer donna en 1901 sur les paysages bibliques. Si Rosegger n'a pas étudié ces œuvres en vue d'un travail scientifique, nous sommes toutefois loin du postulat concernant Ferleitner qui aurait suivi les légendes de sa mère et ses souvenirs pour la rédaction de son manuscrit.

14. - ED 11 p. 201 : «Lebensbeschreibung Jesu Christi, seiner Mutter Maria und vieler Heiligen Gottes. Ein geistlicher Schatz von Pater Cochem».

15. - ED 34 p. 27 : «Jesus Christus war unstreitig der größte Mensch aller Zeiten, und recht hat die Welt, wenn sie ihn ob seines edlen [...] gottähnlichen Geistes den "Gottmenschen" nennt...» ou ED 34 p. 233 : «Unter den Milliarden von Gotteskindern hat der Herr keinen, der ihm so ähnlich wäre, als der Sohn des armen Handwerkerpaares aus Galiläa. Darum ist er sein Einziger, sein Eingeborener».

16. - Cf. ED 38 p. 141.

17. - ED 38 p. 289 : «Und mir hast du Auferstehung gebracht! » schreit Lazar leidenschaftlich vom unteren Ende des Saales her. "Und mir - mehr als das", spricht Maria, feuchten Auges blickt sie auf zu ihm.»

18. - ED 38 p. 246 : «... Laß den Glauben der Väter stehen. Ich weiß ja gleichwohl, daß du es gut meinst, aber andere fassen es nicht und es taugt nimmer, was du tust. Lasse doch die Leute selig werden, wie sie wollen. Sind sie bisher zu Abraham gekommen, so werden sie auch fürder den Weg finden zu ihm - auch ohne deiner».

19. - «Gabriel Mondfels», manuscrit de jeunesse non publié, mais repris partiellement dans «In der Einöde», Preßburg 1872. Dans notre thèse (Peter Rosegger, Poète du terroir perdu, Sorbonne, 1992, également chez Styria, Graz, 1993 : «Peter Rosegger, Dichter der verlorenen Scholle», avec un chapitre sur la religion de Rosegger pourtant moins étoffé), nous avons montré les velléités prophétiques de Rosegger et nous pensons qu'il existe chez lui une assimilation inconsciente à la figure du Christ.

20. - Op. cit., p. 269. Il voit aussi le «Jeune homme riche » qui veut rejoindre le Christ dans le désert, coiffé d'un chapeau orné d'un panache aux plumes rares, de même qu'il lui fait enfourcher - à lui comme à d'autres - un cheval fougueux pour traverser le désert.

21. - Op. cit., p. 162 : « Fabricants de brocs, bourreliers, et bateliers » (?)

22. - Eugénie delle Grazie: «Der 5. Evangelist» «Ein biblischer Roman, wie ihn Ferleitner nie hätte schreiben können und Rosegger nicht sollen». In: Neue Freie Presse 4161, 1905.

23. - ED 38 p. 51 : «So hat der Weise des Ostens die duftenden Gärten Indiens verlassen...»

24. - Op. cit., p. 53 : «Selig werdet ihr sein! Ewig werdet ihr sein!»

25. - ED 9 p. 325 : «Rufe den Menschen... rufe ihn aus der Tierheit von Stufe zu Stufe/ Bis er erwacht vor des Heiligsten Thron / Schauend die Wahrheit im Kranze der Sonnen / Trinkend die Liebe aus feurigen Bronnen -/ Ewig des Ewigen seliger Sohn».

26. - ED 38 p. 211 : «Nichtsein ist nicht».

27. - Op. cit., p. 316 : «In dir wird der Fluch deines Volkes sich erfüllen, herzloser Jude!»

28. - Par exemple : Le 9/60 : « Laisse les morts enterrer leurs morts » ou la parole de Mt 13/12 et Mt 25/29 que Heine parodie : «Hast du viel, so wirst du bald/ Noch viel mehr dazubekommen, / Wer nur wenig hat dem wird /Auch das Wenige genommen. /Wenn du aber gar nichts hast, / Ach, so lasse dich begraben, / Denn ein Recht zum Leben, Lump, / Haben nur, die etwas haben!» 29. - ED 38 p. 105 : «Er denkt nicht, vielmehr es denkt in ihm.»

30. - Op. cit., p. 110 : «Haben sie Durst, so sollen Wasserkrüge herbeigetragen werden... Gott segne es ihnen zum Wein!»

31. - Ibid., p. 111 : «O Vater... Wenn es nach deinem Willen ist, daß aus Wasser Wein wird, so kann es wohl auch sein, daß man frischen Wein in die alten Schläuche gieße, den Geist und die Kraft 
Gottes in den toten Buchstaben!»... «Herr, sagt der Jünger... Schon oft habe ich mir gedacht, du bis anders als wir alle. Du mußt vom Himmel sein.»

32. - Op. cit., p. 116 : «Die Rechte nehmt ihr euch selbst und die Pflichten werden euch gegeben. Des Weibes Gebot ist: Du sollst nicht ehebrechen!»

33. - Hérode Antipas fut seulement tué en 36 ap. J.-C. par Arétas.

34. - ED 38 p. 143 : «Erst seit wenigen Wochen verheiratet mit dem alten braven Jobsohn, dem Freunde ihrer Eltern. Hintergeht ihn und läuft einem jungen Fant nach!»

35. - Op. cit., p. 249 : «Saul, der grimme Weber».

36. - Op. cit., p. 207 : «Ja, meine Freunde, eher geht das Kamelhaar durch ein Nadelöhr, als der Reiche in unseren Himmel».

37. - Ici il ne s'agit pas d'une affabulation, mais probablement d'une lacune biblique de Rosegger. L'âne sur lequel le Christ doit entrer dans Jérusalem a été deux fois pré-dessiné dans l'Ancien Testament: dans Moïse 4/20, et notamment dans Zacharie 9/9: «Éclate de joie, Jérusalem ! Pousse des acclamations, ville de Zion! Regarde, ton Roi vient à toi, juste et victorieux, humble et monté sur un âne, sur un ânon, le petit d'une ânesse... »

38. - C'est le vieux sage Steinklopferhanns dans la comédie «Die Kreuzelschreiber» qui dit: «... und hast du bisher's ganze Pfund glaubt, werdn dich die paar Lot Zuwag a nit umbringen!» (Akt I, Szene 6)

\section{RÉSUMÉS}

À une époque où la recherche sur ce que pouvait être la « véritable histoire du Christ » avait déjà presque tout dit avec David Friedrich Strauß et Ernest Renan, Peter Rosegger (1843-1918) poète styrien, autodidacte, et néanmoins prophète en son pays, veut, en 1905 avec son roman «I.N.R.I. - la Bonne Nouvelle apportée par un pauvre pécheur ", se redonner l'innocence des Évangélistes. Il prend la plume sous le déguisement d'un condamné à mort qui, pour tromper son angoisse en attendant l'exécution capitale, n'a que sa mémoire pour réécrire la vie du Christ, et produit alors ce qu'un critique de l'époque appela "Le $5^{\mathrm{e}}$ Évangile ", interprétation iconoclaste et haute en couleur des événements bibliques, étonnante de la part d'un homme qui se dit croyant et catholique.

$\mathrm{Zu}$ einer Zeit, wo über das wahre Leben Christi mit David Friedrich Strauß und Ernest Renan schon fast alles gesagt schien, macht sich der Waldbauernbub und Prophet aus der Steiermark daran, mit seinem Roman «INRI - die Frohe Botschaft eines armen Sünders» das Evangelium neu zu schreiben. In der Gestalt eines aus politischen Gründen zum Tode Verurteilten, der sich das bange Warten vor der Hinrichtung erleichtern will, greift er zur Feder, um sich, angeblich nur auf sein Gedächtnis gestützt, das Neue Testament nachzuerzählen. Was dabei herauskommt, ist ein erstaunliches Gemisch von «Dichtung und Wahrheit», angesichts dessen Rosegger als «fünften Evangelisten» jede Rechtgläubigkeit abgesprochen werden müßte.

\section{AUTEUR}

\section{EVA PHILIPPOFF}

Université Charles-de-Gaulle - Lille III 